\title{
Wave-particle interaction effects in the Van Allen belts
}

\author{
Daniel N. Baker ${ }^{*}$ (1)
}

\begin{abstract}
Discovering such structures as the third radiation belt (or "storage ring") has been a major observational achievement of the NASA Radiation Belt Storm Probes program (renamed the "Van Allen Probes" mission in November 2012). A goal of that program was to understand more thoroughly how high-energy electrons are accelerated deep inside the radiation belts_-and ultimately lost—due to various wave-particle interactions. Van Allen Probes studies have demonstrated that electrons ranging up to 10 megaelectron volts (MeV) or more can be produced over broad regions of the outer Van Allen zone on timescales as short as a few minutes. The key to such rapid acceleration is the interaction of "seed" populations of 10-200 keV electrons (and subsequently higher energies) with electromagnetic waves in the lower band (whistler-mode) chorus frequency range. Van Allen Probes data show that "source" electrons (in a typical energy range of one to a few tens of keV energy) produced by magnetospheric substorms play a crucial role in feeding free energy into the chorus waves in the outer zone. These chorus waves then, in turn, rapidly heat and accelerate the tens to hundreds of keV seed electrons injected by substorms to much higher energies. Hence, we often see that geomagnetic activity driven by strong solar storms (coronal mass ejections, or CMEs) commonly leads to ultra-relativistic electron production through the intermediary step of waves produced during intense magnetospheric substorms. More generally, wave-particle interactions are of fundamental importance over a broad range of energies and in virtually all regions of the magnetosphere. We provide a summary of many of the wave modes and particle interactions that have been studied in recent times.
\end{abstract}

Keywords: Plasma waves, Energetic particles, Radiation belts, Acceleration

\section{Introduction}

It is often asserted that the Earth's near-space environment is a vast plasma physical laboratory. Key earlier papers have examined in considerable theoretical depth the principal wave modes likely affecting electron acceleration and loss in the radiation belts (Summers et al. 2007). Perhaps no spacecraft mission has more clearly or capably been able to examine experimentally the waveparticle interactions than NASA's Radiation Belt Storm Probes (RBSP) program. The dual-satellite RBSP vehicles were fully instrumented to measure magnetic fields, electric fields, plasma electrons and ions, and a full spectrum

*Correspondence: daniel.baker@lasp.colorado.edu Laboratory for Atmospheric and Space Physics, University of Colorado Boulder, 3665 Discovery Drive, 600 UCB, Boulder, CO 80303, USA of radiation belt energetic particles. The state-of-the-art sensors were geared toward understanding deeply how radiation belt particles were accelerated, transported, and lost from the Van Allen belt regions surrounding the Earth (Mauk et al. 2013).

The RBSP spacecraft were instrumented with identical sensors onboard the two vehicles. The satellites were launched together into a geostationary transfer orbit (GTO) on 30 August 2012. Only about 2 days after the successful launch and RBSP orbit insertion, the scientific sensors began to be switched on. Notably, the Relativistic Electron-Proton Telescopes (Baker et al. 2013a) on both the RBSP-A and RBSP-B spacecraft began acquiring data on 1 September 2012. Almost immediately, the Relativistic Electron-Proton Telescope (REPT) sensor made new discoveries about the morphology of the outer Van 
Allen radiation belt (Baker et al. 2013b). The detection and characterization of the so-called relativistic electron "storage ring" (or third Van Allen belt) was the beginning of a whole series of new discoveries and insights into the structure and dynamics of the radiation regions shrouding our planet. The understanding that came from the RBSP data continued at an impressive pace from the beginning of 2012 until the two RBSP spacecraft ran out of station-keeping fuel in 2019 and the mission was brought to an operational end (Baker et al. 2021).

In this paper, we will focus on wave-particle effects that were studied using the comprehensive RBSP data sets. Much of our attention will be directed toward the understanding gained about relativistic and ultra-relativistic electrons $(E \sim 1 \mathrm{MeV}$ to $E>10 \mathrm{MeV})$ in the outer Van Allen radiation zone. Our review will discuss extensions of earlier groundbreaking work (Meredith et al. 2003) showing the central role of chorus waves in high-energy electron production in the outer zone. We will review measurements from the REPT sensors used in conjunction with plasma, fields, and lower energetic particle sensors to portray several of the mechanisms that are at play in the Earth's magnetosphere. In doing so, we will examine a number of wave modes and their effects.

\section{Interplanetary shock effects and MHD waves}

Events that modulate or accelerate energetic particle fluxes in the magnetosphere on very large scales are often driven by the interplanetary shock waves. Such shocks striking the magnetosphere can produce strong transient electric field pulses that propagate rapidly through the entire magnetosphere (Wilken et al. 1982; Foster et al. 2015; Kanekal et al. 2016). Under some circumstances, an interplanetary shock hitting the magnetosphere can produce a local depletion of energetic electron fluxes, while in many other cases, there can be rapid, powerful flux enhancements extending up to multi-megavolt energies (Schiller et al. 2017). Strong magnetohydrodynamic (MHD) waves produced by shock sudden impulse impacts on the magnetosphere can produce long lasting "ringing" of broad regions of the inner magnetosphere.

Figure 1 shows an example of a strong shock wave that struck the magnetosphere at 1540 UT on 31 October 2012. The RBSP-A spacecraft was near apogee (geocentric radial distance $r \sim 5.8$ Earth radii, $R_{\mathrm{E}}$ ) at the time of the impact and was near the dawn meridian in magnetic local time (MLT). Figure 1 shows one 9-h orbit's worth of RBSP-A data from perigee at 1200 UT to the next perigee at $2100 \mathrm{UT}$ on 31 October. The REPT data from several electron channels in the figure (ranging from $E=1.8 \mathrm{MeV}$ to $E=6.95 \mathrm{MeV}$ ) show that the shock impact produced a clear oscillatory wave pattern in the flux time profiles extending up to at least $E \sim 5.0 \mathrm{MeV}$. Effects were seen to even higher energies $(E=5.6$ and $E=6.95 \mathrm{MeV})$ but the oscillatory behavior was masked by statistical fluctuations in the counting rates of the highest channels. The dominant effect in the electron fluxes appeared to be an initial reduction in the flux (for $1.8-4.5 \mathrm{MeV}$ electrons) followed by a periodic recovery of the flux. The periodic wave modulation of fluxes lasted for well over an hour with more rapid flux oscillations (higher frequencies) at the higher energies. At the highest energy shown in

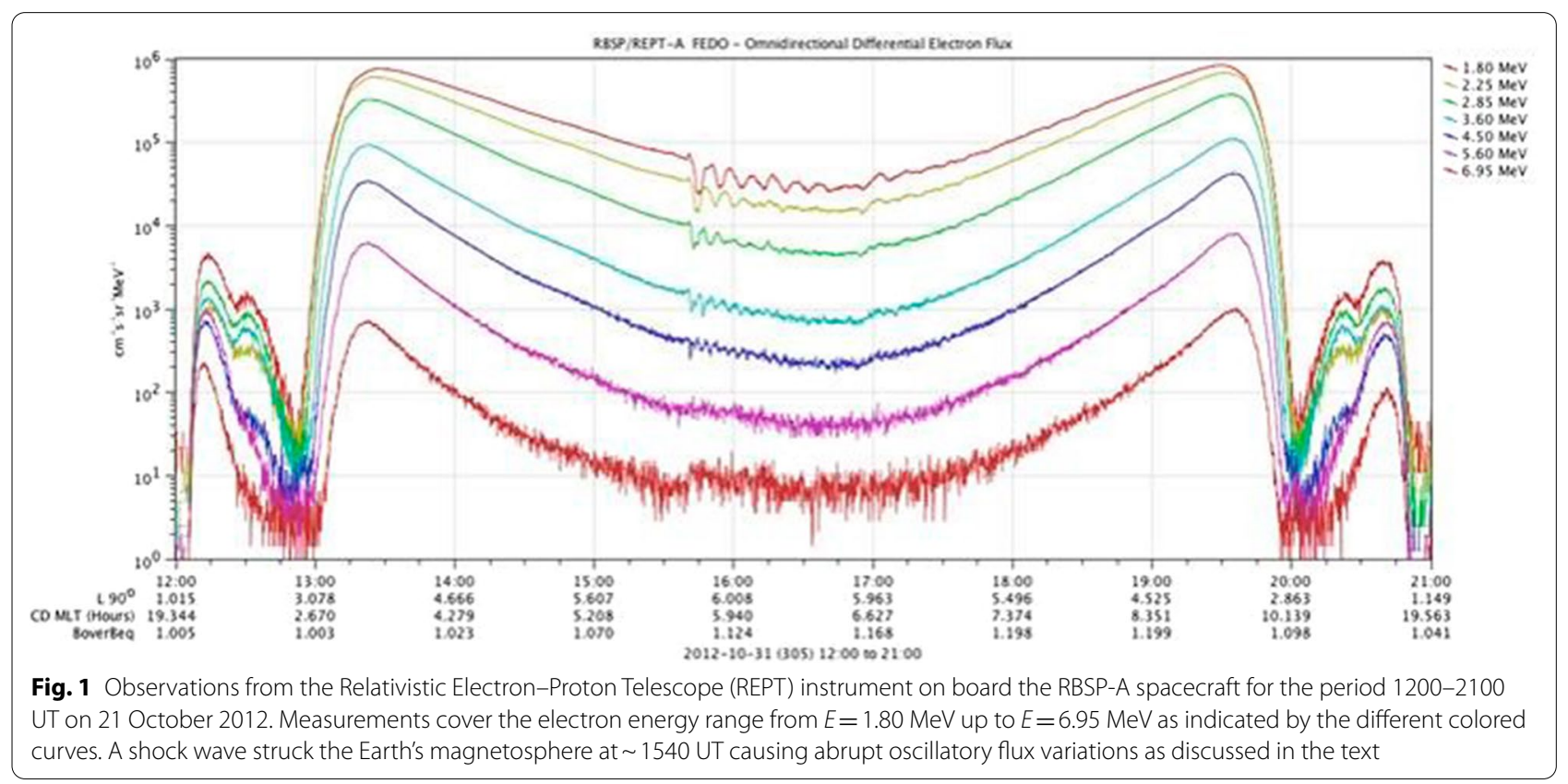


Fig. 1, the flux of electrons increased quite visibly as a result of the shock impact.

More comprehensive observations of particle and field signatures for a strong shock event were presented by Foster et al. (2015). At 2021 UT on 8 October 2013, a powerful IP shock struck the magnetosphere. The event was observed not only by the RBSP-A and -B spacecraft, but also by sensors on board the several THEMIS spacecraft as well (Foster et al. 2015). The RBSP-A spacecraft was deep inside the magnetosphere at $r \sim 3 R_{\mathrm{E}}$ at the time of the shock impact, while RBSP-B was at higher altitude near $r \sim 4.5 R_{\mathrm{E}}$. Both RBSP spacecraft were in the afternoon LT sector.

Figure 2 shows in the upper two panels the azimuthal electric field profiles for RBSP-A and RBSP-B, respectively, for the 1-h period 2000-2100 UT. The large-amplitude electric field pulse resulting from the shock impact was evident at both spacecraft locations (with a very slightly earlier-in-time signal for the "A" spacecraft). The lower two panels of Fig. 2 show the pitch angle resolved energetic electron data from the identical $E=3.6 \mathrm{MeV}$ channels of the REPT-A sensor (third panel) and the REPT-B sensor (fourth panel). Note the very prompt enhancement of $90^{\circ}$ pitch angle electrons for REPT-B as soon as the shock wave hit the magnetosphere. There then were several subsequent "drift echo" enhancements as the initial population of electrons produced by the shock impact drifted completely around the Earth multiple times over the next 40 or so minutes (Foster et al. 2015). The REPT-A sensor also saw drift echo signatures of $E=3.6 \mathrm{MeV}$ electrons after 2030 UT due to the shock event, but did not see the electron enhancement as promptly as the "B" spacecraft. Clearly, the strength and timing of such shock effects depends on local time and radial locations of the observing platforms. In fact, for this (and many other) IP shock events during the

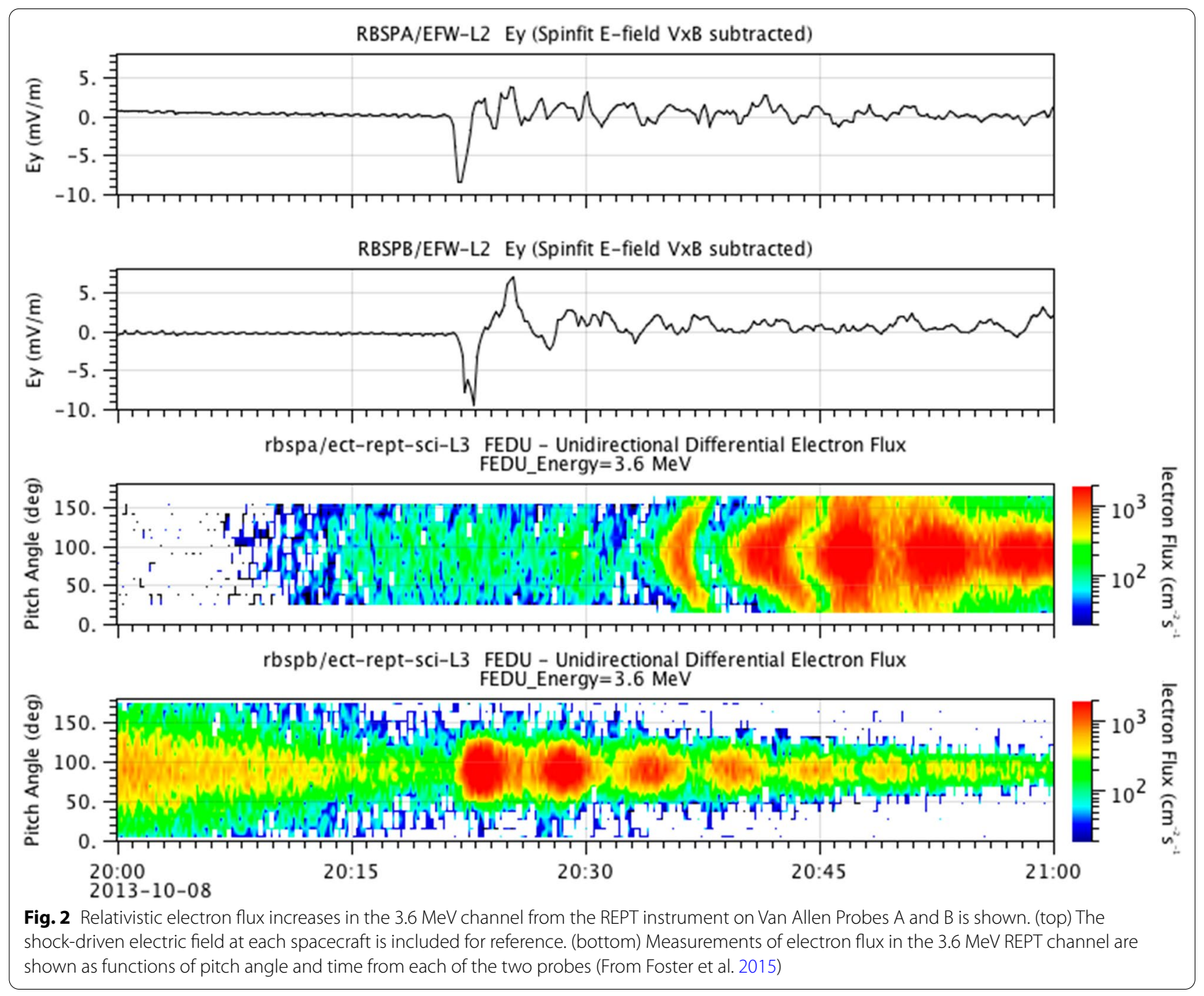


RBSP (or "Van Allen Probes") era, shock impacts produced rapid flux enhancements to energies well above $E=6 \mathrm{MeV}$ (Foster et al. 2015; Kanekal et al. 2016; Schiller et al. 2017). Clearly these types of events are global scale MHD wave-related disturbances.

\section{Higher frequency plasma wave phenomena}

The Van Allen Probes had onboard sensors capable of measuring wave phenomena across the entire spectrum of frequencies and wavelengths relevant for radiation belt particle acceleration and loss. An illustration of this is shown in Fig. 3. This portrays RBSP-B combined data for low and medium energy electrons, electric field data, and plasma wave data for a representative day (19 September 2012) shortly after the RBSP launch. Note also that the times of plasmapause crossings (as inferred from the field and plasma wave data) are also indicated by the vertical dashed lines.

The upper panel of Fig. 3 shows color-coded (log flux) energy-time intensities of $E \sim 40$ to $E \sim 200 \mathrm{keV}$ electrons for RBSP-B from the low-range head of the MagEIS instrument (Blake et al. 2013). Several magnetospheric substorm injection events were evident around times when RBSP-B was near apogee. The second panel of Fig. 3 shows the inferred spacecraft charging level over time based on Electric Field and Waves (EFW) measurements (see Wygant et al. 2013). A large charging event reaching a potential near $40 \mathrm{~V}$ was seen around $2000 \mathrm{UT}$ associated with the strong substorm injection seen in the MagEIS data as show in the top panel.

The third panel of Fig. 3 shows wave data from a few Hertz (Hz) to $\sim 10 \mathrm{kHz}$. Such measurements reveal lowfrequency (Alfven wave) activity as well as plasmapheric hiss waves (around $100 \mathrm{~Hz}$ ), magnetosonic waves (just outside the plasmapause as labelled), and broadband whistler waves that are most intense during substorm injections of low and moderate energy electrons (see Panel 1). The fourth and fifth panels of Fig. 3 confirm the wave identifications by showing EMFISIS search coil data (Kletzing et al. 2013) and by showing (in the fifth panel) the ratio of $\mathrm{E} / \mathrm{B}$ field values. The ratio $\mathrm{E} / \mathrm{B}$ indicates the strength of electromagnetic modes for whistler waves and gives clear identification of hiss, magnetosonic, and Alfven wave activity. Clearly, the RBSP sensor provide comprehensive wave measurements throughout the inner magnetosphere.
Such low-energy particle measurements and wave measurements, as shown in Fig. 3, proved indispensable for study of specific radiation belt enhancement events. A notable example of such a powerful radiation belt enhancement event occurred on 17 March 2013. A wellobserved solar coronal mass ejection (CME) occurred on 15 March 2013. This CME had associated with it a sharply defined shock wave and this struck the Earth's magnetosphere at 0604 UT on 17 March (Baker et al. 2014a). The outer radiation belt was rapidly depleted of multi-MeV electrons following the shock impact.

As analyzed in detail by Foster et al. (2014), the radiation belt electron population from $\sim 1$ to $\sim 10 \mathrm{MeV}$ remained quite depleted from 0600 UT until $\sim 2200$ UT on 17 March. Then, at $\sim 2200$ UT a strong magnetospheric substorm was observed to occur. Figure 4 (from Foster et al. 2014) shows in the middle panel that there was a strong and abrupt energetic $(\sim 50 \mathrm{keV})$ electron injection observed by the MagEIS-A sensor (blue trace). Concurrently (green curve), the local plasma density measured by EMFISIS dropped precipitously. These are classic signatures of substorm onset (Baker et al. 1996). The lower panel of Fig. 4 shows also that the magnetic field local to RBSP-A increased rapidly as part of the magnetic field "dipolarization" that characterizes the substorm onset (Baker et al. 1996).

The top panel of Fig. 4 shows that concurrent with the $E \sim 50 \mathrm{keV}$ electron injection of the substorm, there was an immediate and very distinct enhancement of the VLF chorus waves measured by EMFISIS sensors. Almost immediately (see the lower panel) the $E=2.85 \mathrm{MeV}$ electrons measured by the REPT-A sensors began a rapid recovery. As shown by Foster et al. (2014, 2017), substorm events and the associated chorus wave enhancements can rapidly and impressively replenish the entire depleted outer Van Allen radiation zone on a time scale of a few tens of minutes. As further studied by Omura et al. (2019), the nonlinear wave-particle interactions during events such as that on 17 March 2013 through oblique chorus wave interactions can greatly accelerate highly relativistic electrons on time scales of just a few minutes. This demonstrates that Earth's magnetosphere is a remarkably efficient and effective electron accelerator that uses wave power from storm-generated rising-tone chorus bursts (see Meredith et al. 2003) to accelerate

(See figure on next page.)

Fig. 3 Collection of data from several instruments on board the RBSP-B spacecraft on 19 September 2012. The upper panel shows an energy-time spectrogram (color-coded) for the entire day, while the second panel shows the level of spacecraft charging inferred for the same period. Panels 3,4 , and 5 show various wave modes identified from the RBSP sensors (as described in the text). The lower panel shows the spacecraft geocentric radial distance as a function of time 

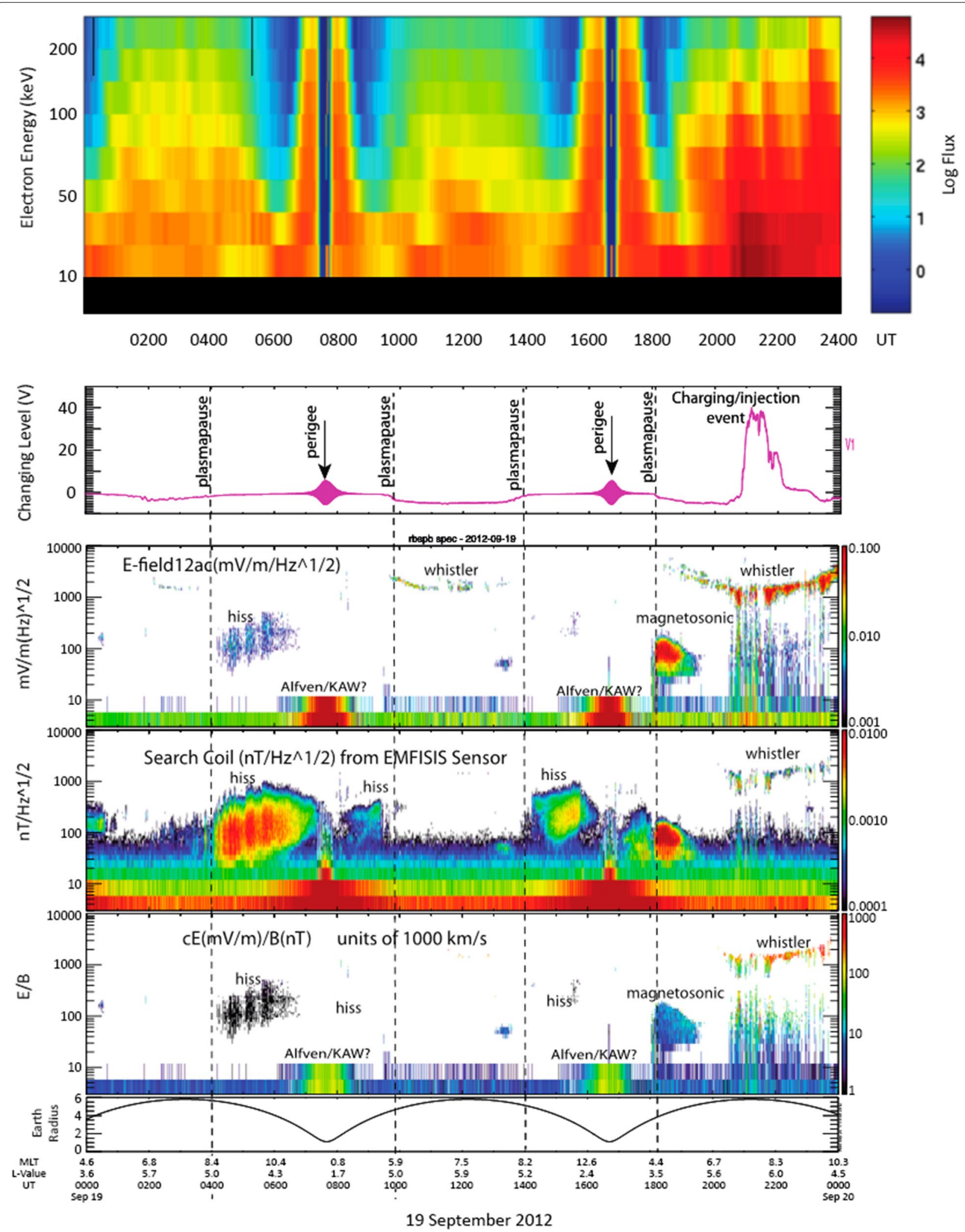

Fig. 3 (See legend on previous page.) 


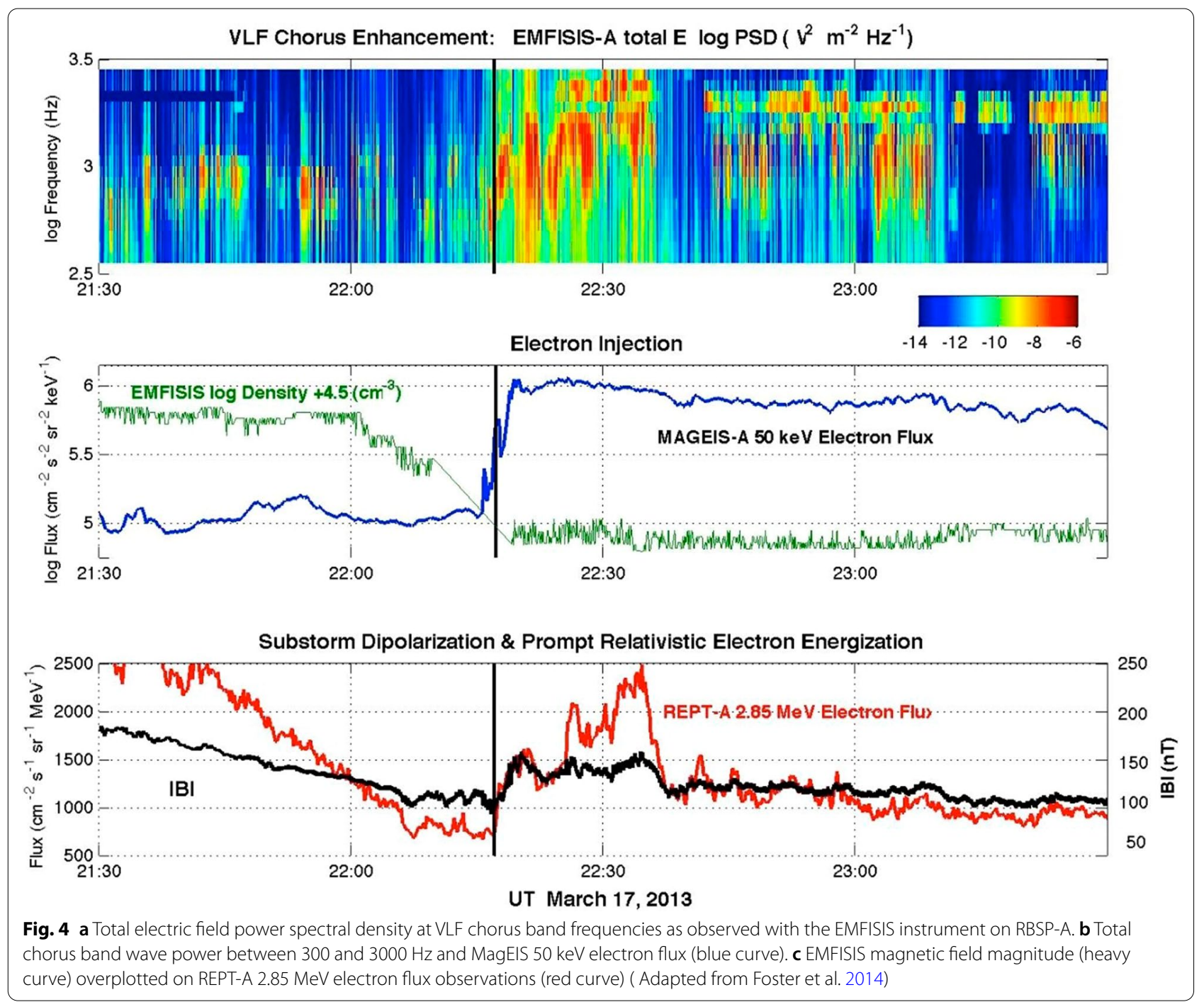

extremely high-energy electron populations in the heart of the outer Van Allen belt.

\section{Radiation belt structure and dynamics}

As remarked upon in the prior section of this paper, strong CME-induced changes of the near-Earth solar wind environment can alter dramatically the large-scale configuration of the radiation belts. As shown by Van Allen Probes studies (Baker et al. 2013b, 2014a, 2016, 2019), solar wind changes caused by CME events can first greatly deplete the vast majority of the outer Van Allen zone electron population and then subsequent geomagnetic storm processes (see, also, Miyoshi et al. 2013) can rapidly replenish the outer belt region (as discussed in prior section). The Van Allen Probes proved to be ideal tools for studying the global-scale morphology changes of the radiation belts from such major solar events over periods of days, weeks, and months. In this section we will examine some of these kinds of broad radiation belt configuration changes.

Previously we described the detailed behavior of the outer zone electrons for the specific time that outer radiation belt was depleted (Baker et al. 2014a) and for the time when the outer belt was rapidly repopulated (Foster et al. 2014) during the March 2013 CME-driven storm event. In a subsequent study of the "St. Patrick's Day" storm of March 2013, Baker et al. (2019) examined the more global features of this same storm event. Here, Fig. 5 shows the globally mapped outer belt configuration for $E=4.2 \mathrm{MeV}$ electrons throughout the day on 17 March 2013.

The patterns shown in Fig. 5 are derived using the electron flux measurements along the elliptical orbital trajectories for the RBSP-A and RBSP-B spacecraft. Careful examination of each panel of Fig. 5 shows the trajectory of the RBSP-A spacecraft projected onto the magnetic 


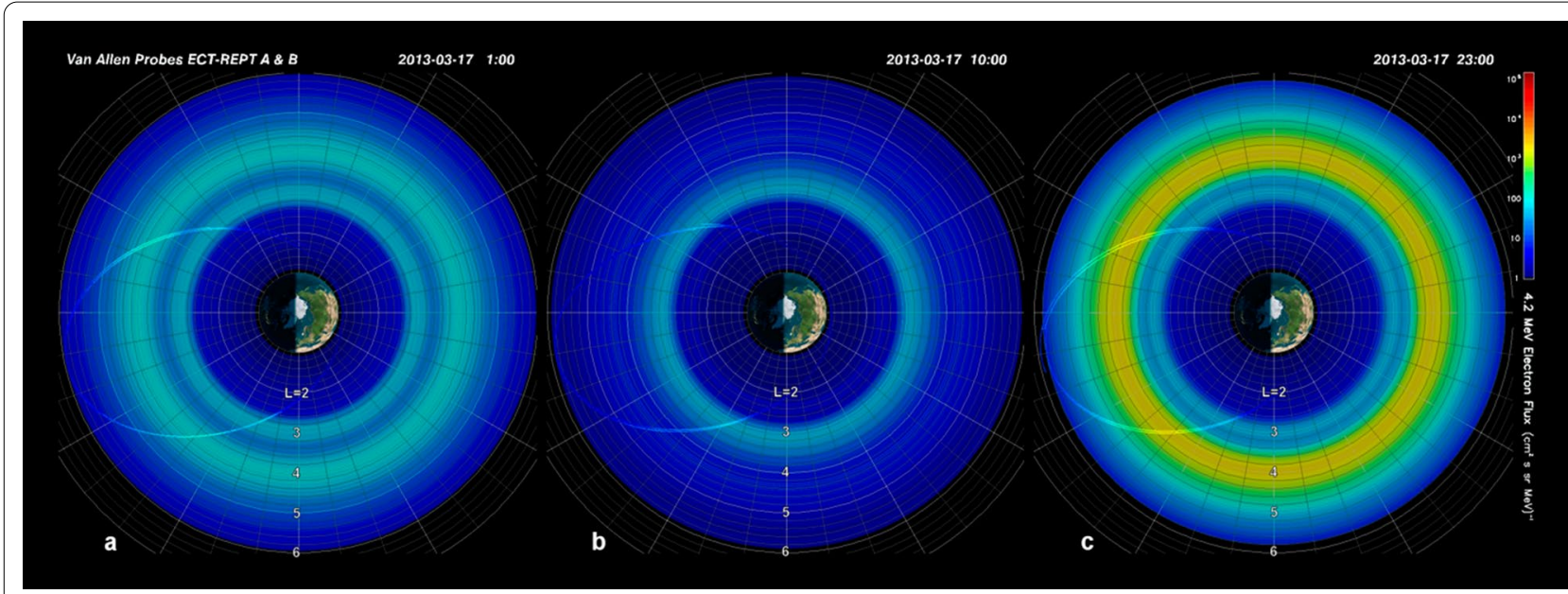

Fig. 5 Polar plots of electron fluxes for 4.2-MeV channel for 17 March 2013. a Pattern for 0100 UT. b For 1000 UT. c For 2300 UT for times shown (from REPT = Relativistic Electron-Proton Telescope) (From Baker et al. 2019)

equatorial plane. The flux of $4.2 \mathrm{MeV}$ electrons at each point along the orbital trajectory is color-coded according to the logarithmic intensity bar to the far right of the figure. Since the electrons measured by the REPT sensors drift azimuthally around the Earth on magnetic drift shells much faster than the spacecraft cuts across $L$-shells, it is sensible (and reasonable) to map the locally measured fluxes all the way around the Earth to indicate the distribution of electrons throughout the entire radiation belt region (Baker et al. 2019).

In Fig. 5a for belt passages early on 17 March (before the IP shock passage at 0600 UT), the outer Van Allen belt had a striking double-peaked structure. The "normal" outer belt stretched from $L \sim 3.5$ out to about $L=5.0$. However, the electron "storage ring" feature (Baker et al. 2013b) was clearly visible from $L \sim 2.8$ out to $L \sim 3.2$. When one realizes that there also was an inner zone proton belt at the same time (Selesnick et al. 2016), the 17 March pre-storm conditions were a clear example of the three-belt radiation configuration.

In Fig. 5b, the data are shown for the times around 1000 UT on 17 March 2013. This was after the CMEdriven shock wave had struck the magnetosphere thereby depleting much of the outer zone. As is evident from the figure, only the storage ring electron population escaped loss and so electrons from $L \sim 3$ to $L \sim 3.5$ remained trapped, while all other outer zone electrons were lost. As described by Baker et al. (2016), many of the electrons at $L \gtrsim 4.0$ were probably lost outward through the magnetopause, but those closer to the earth $(L \lesssim 4.0)$ were probably scattered by whistler waves into the atmospheric loss cone.
Figure $5 \mathrm{c}$ shows $E=4.2 \mathrm{MeV}$ data for the period late on the day of 17 March 2013. This is after the strong substorm activity studied by Foster et al. (2014). By this time, the outer Van Allen belt was broadly replenished and much enhanced over the pre-storm electron intensities. The outer zone was wider in radial extent than prior to the shock impact and the outer belt was orders of magnitude higher in absolute fluxes. However, note that the storage ring (third belt) was about the same in position and strength throughout this event period.

An event having many similarities to the March 2013 storm period occurred almost exactly 2 years later in March 2015. This 2015 St. Patrick's Day event was compared and contrasted with the 2013 event in the papers by Baker et al. (2016, 2019). Figure 6 shows several types of Van Allen Probes (RBSP-A) data for the period of 0315 UT to 0500 UT on 19 March 2015. The upper panel shows REPT-A electron data for the $E=2.1 \mathrm{MeV}$ channel. The data are shown as color-coded pitch-angle resolved electron intensities versus time. The second panel (Fig. 6b) shows electron data for the $E=231 \mathrm{keV}$ MagEIS channel in a format similar to that in Fig. 6a. Finally, Fig. 6c (lower panel) shows the energy-time spectrogram (also color-coded) for the RBSP-A HOPE oxygen channels.

Marked on the Fig. 6 panels by the black vertical dashed lines is an indication of the plasmapause location (inferred from the concurrent EFW and EMFISIS wave sensors). Also shown by the blue vertical dashed line is the innermost extent of the highly relativistic electrons as measured by REPT-A. (This is termed the "Impenetrable Barrier" and will be discussed further below). As is clear in Fig. 6a, no discernable flux of $E=2.1 \mathrm{MeV}$ electrons 


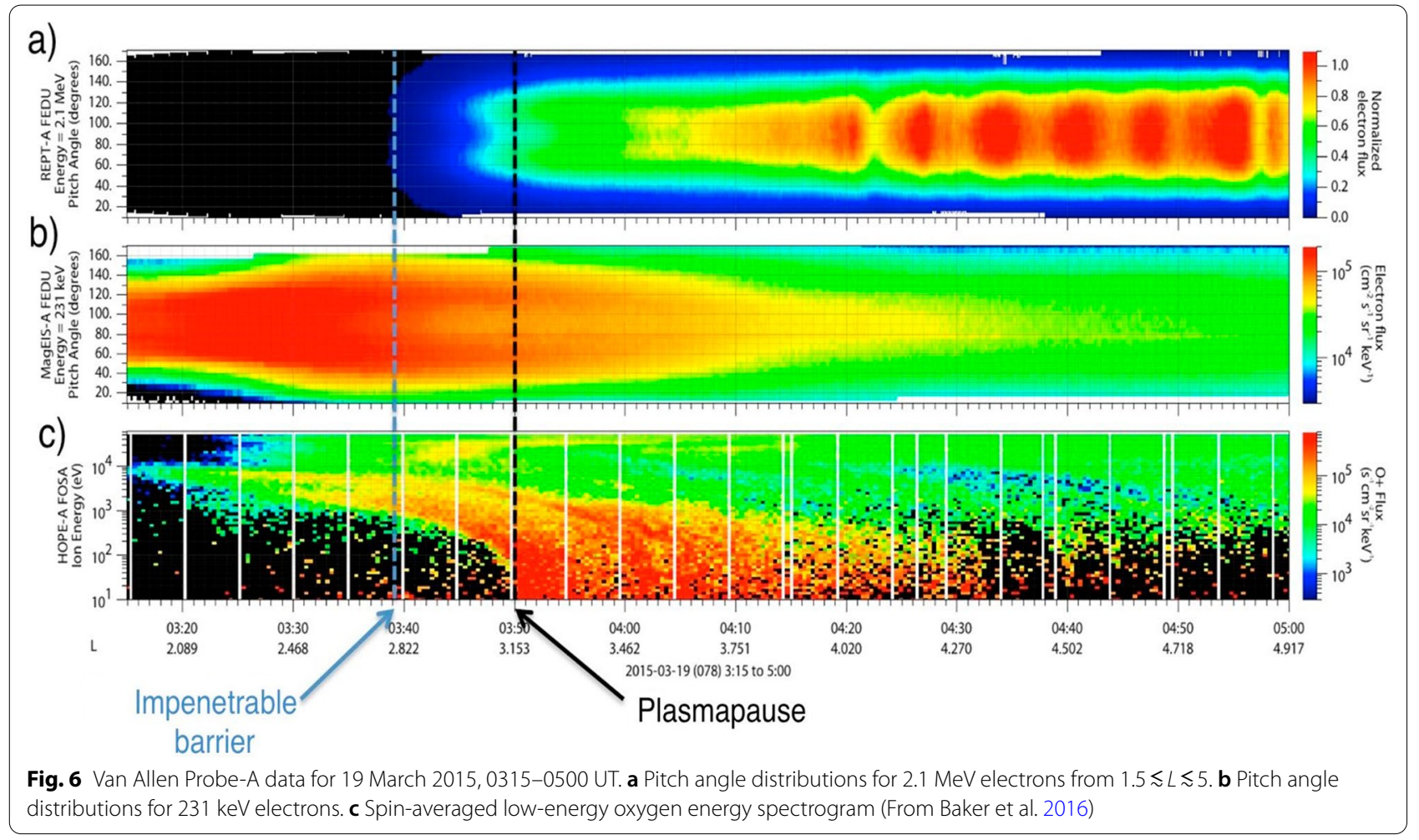

was observed inside of $L \sim 2.8$ on this post-storm day. Near and just inside the plasmapause, these electrons had a clear "butterfly" (bimodally peaked) pitch angle distribution. As described by Baker et al. (2016), this double-peaked distribution may have been due to local acceleration associated with magnetosonic waves interacting with lower energy (hundreds of $\mathrm{keV}$ electrons) near the plasmapause (reference Fig. 3).

Note in Fig. 6 that no multi-MeV electrons were seen to move inside of $L=2.8$. This same fact was true in the March 2013 event period (see Fig. 5). However, the hundreds of keV electrons measured by MagEIS (Fig. 6b) were able to penetrate freely inward to $L \lesssim 3$ and seemed unfazed by the plasmapause boundary. In addition, as shown in Fig. 6c, energetic oxygen $\left(\mathrm{O}^{+}\right)$ions were able to penetrate deeply into the inner magnetosphere in considerable contrast to the high-energy electrons. After $\sim 0400$ UT on this day-and in the outer part of the outer Van Allen zone-there were high fluxes of $E=2.1 \mathrm{MeV}$ electrons $(L \gtrsim 3.5)$ showing strongly trapped $\left(90^{\circ}\right.$ pitch angle) electrons.

The March 2015 storm period produced some of the strongest relativistic electron enhancements during the entire Van Allen Probes era (see Baker et al. 2016, 2019). As such there were strong enhancements from $L \sim 2.8$ throughout the outer magnetosphere out to (and beyond) the geostationary orbit $(L \sim 6.6)$. Fluxes of electrons increased significantly up to energies above $10 \mathrm{MeV}$ during the main storm period of 17-20 March 2015 (see Baker et al. 2019).

Following the storm interval itself, the energetic electrons near the heart of the outer radiation zone showed fascinating evolution of the overall electron energy spectrum. This was studied in detail by Zhao et al. (2019a). Figure 7 shows combined data from the MagEIS and REPT sensors for the period 20 March to 30 March. The upper row of panels show energy spectra for electrons from $100 \mathrm{keV}$ to $10 \mathrm{MeV}$. The left most panel is for $L=3.0$. The middle column of Fig. 7 is for $L=3.5$ and the right column is for $L=4.0$. The color-coding shows the data of each energy spectrum with deep blue corresponding to 20 March all the way up to bright red corresponding to spectra taken on 30 March.

As described in the study of Zhao et al. (2019a) the overall electron energy spectra acquired around 20 March were a "broken" power law with a hard spectrum from $100 \mathrm{keV}$ to about $E=2 \mathrm{MeV}$ and then a steeper spectrum from $2 \mathrm{MeV}$ up to $10 \mathrm{MeV}$. This spectral shape applied across the range from $L=3$ or to beyond $L=4$. However, as time progressed from 20 March forward, the fluxes of electrons from $\sim 200 \mathrm{keV}$ to $\sim 1 \mathrm{MeV}$ began to diminish substantially. By 30 March (as shown by the reddish spectral traces) there was a dramatic diminution of medium energy electrons even though the electron 

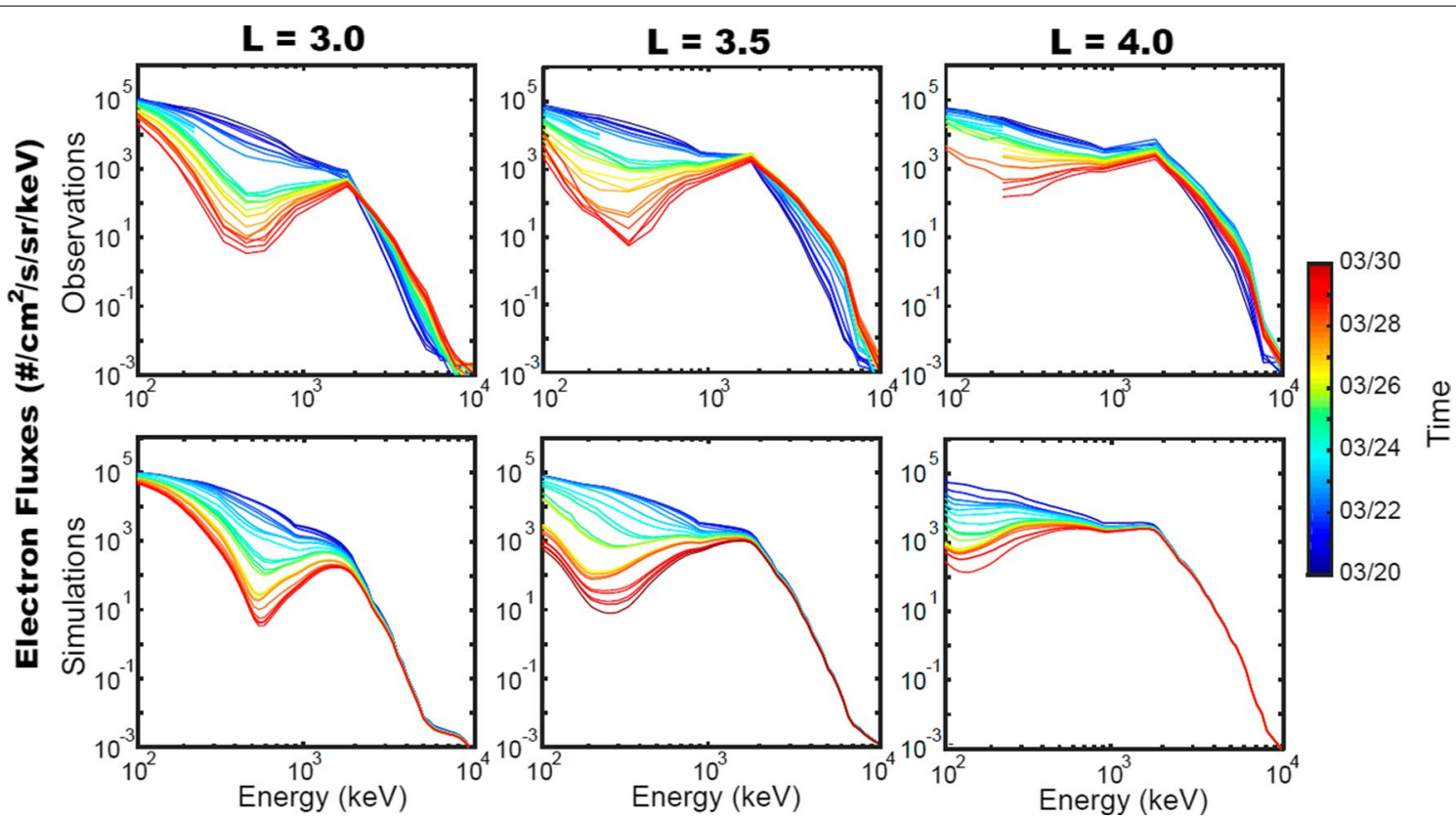

Fig. 7 Comparison of Fokker-Planck simulations (bottom row of three panels) with observations of energetic electron spectra made in March 2015 by the RBSP spacecraft. The color coding indicates the dates when data were acquired from 20 to 30 March 2015 ( Adapted from Zhao et al. 2019a)

fluxes above $E \sim 1 \mathrm{MeV}$ were hardly changed. This led to what Zhao et al. termed a "reversed" energy spectrum.

Using observed wave data from the EMFISIS sensors onboard the RBSP spacecraft, Zhao et al. modeled the interaction of hiss waves with the initial (20 March) electron energy spectrum. The lower row of panels in Fig. 7 show the modeled evolution of the energy spectra at the several L positions as a function of time. Clearly, these results show that hiss waves acting to scatter (and thus precipitate) hundreds of $\mathrm{keV}$ electrons explains almost perfectly the spectra seen for energetic electrons after the 2015 St. Patrick's Day storm. Also quite clearly, the hiss waves were expected to have little effect on the multi$\mathrm{MeV}$ electron population.

\section{Anthropogenic effects on the radiation belts}

One of the most striking and persistent morphological features seen in the multi-MeV data from the Van Allen Probes was the sharp inner extent of the outer radiation belt. This feature is evident in Figs. 5 and 6 shown above and was the precise focus of a dedicated paper by Baker et al. (2014b). As commented previously in this review, Baker et al. (2014b) termed the sharp inner edge of the outer Van Allen belt as the "impenetrable barrier", because even during strong solar wind forcing during the RBSP era, multi-MeV electrons were never seen to move inward of an equatorial radial distance of $r \sim 2.8 R_{\mathrm{E}}$. This $L \sim 2.8( \pm 0.1)$ inward extent of the relativistic electrons seemed to persist, often for weeks or months at a time.
In the original study, Baker et al. (2014b) attributed the "barrier" to natural effects, where increasing geomagnetic field strengths and diminishing pitch angle diffusion would slow any inward transport of very energetic electrons. In this picture, it simply was a natural consequence of greatly slowed inward radial diffusion that the barrier always appeared right around $L=2.8$.

In subsequent studies, however, the REPT team studied the impenetrable barrier from a different perspective (Foster et al. 2016, 2020). In this later work, it was recognized that plasma waves tended to be ducted along magnetic field lines (especially inside the plasmasphere) and can represent the presence of quite high wave power relatively close to the Earth. Figure 8 illustrates notionally that waves triggered by mid-latitude lightning or from powerful human transmitters can populate a Very Low Frequency (VLF) "bubble" (Foster et al. 2016).

The Van Allen Probes sensors proved to be very useful for studying the VLF bubble properties. Figure 9 shows EMFISIS high-frequency receiver data for a selected day (8 October 2013). The data presented in the upper panel show a frequency vs. $L$ value color spectrogram for the HFR instrument. The smooth downward sweeping red curve in Fig. 9a is the value of one-half of the electron gyrofrequency ( $1 / 2 \mathrm{fce})$ based on the magnetic field strength across the shown $L$ value range. The color-coded data generally lying below and to the left of the $1 / 2$ fce trace are the wave powers detected by the HFR sensor. As is clear from the color bar to the right of the panel, 


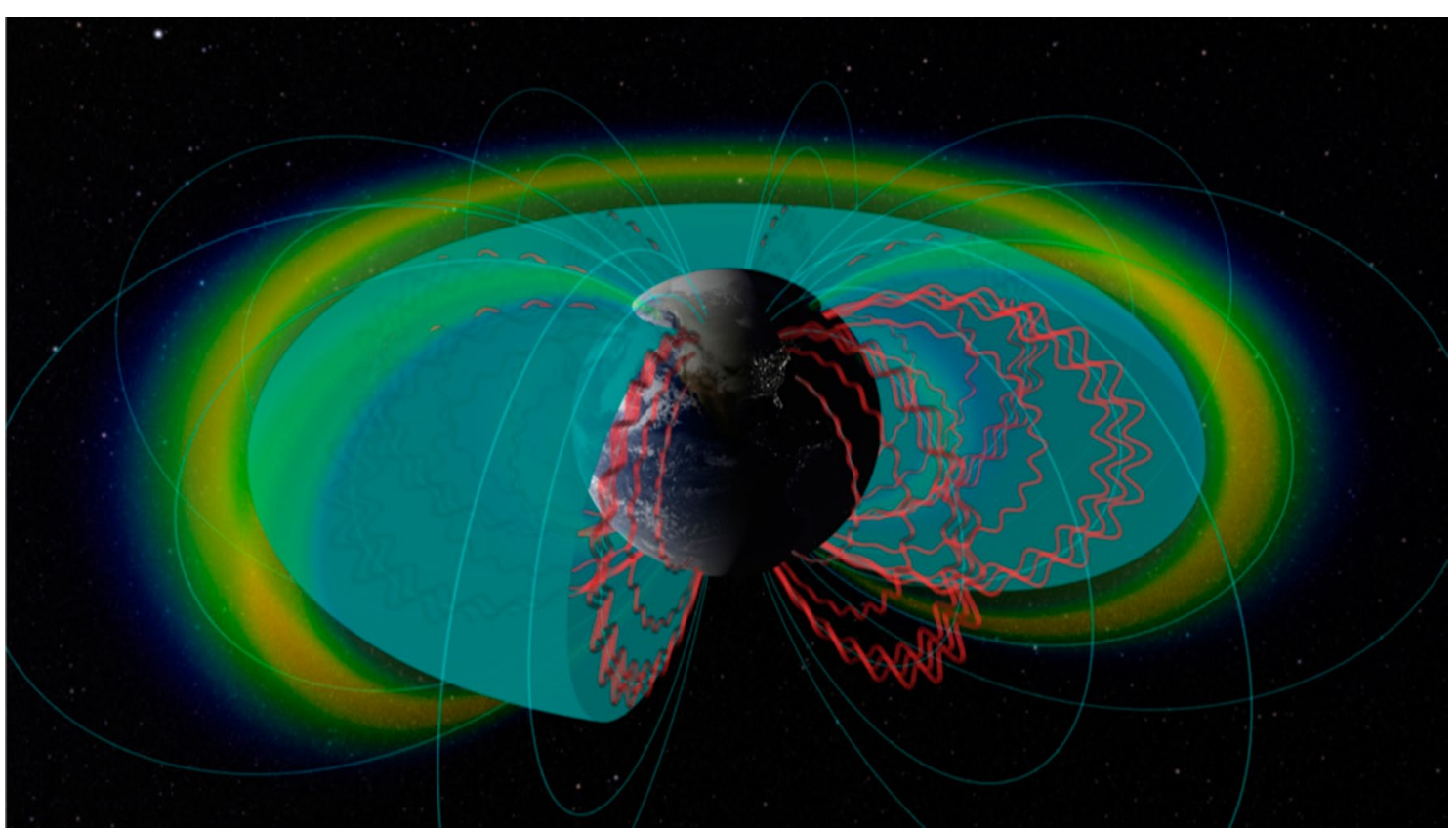

Fig. 8 Schematic diagram illustrating the inner part of Earth's magnetosphere. The outer edge of the VLF "bubble" is sketched showing intense waves confined inside this boundary (Courtesy of NASA)

there was mid-level broadband power across most of the frequency range below $1 / 2$ fce. However, there were also very intense power levels at discrete frequencies ranging from $\sim 20 \mathrm{kHz}$ on up to $\sim 65 \mathrm{kHz}$. As noted by Foster et al (2016), the discrete frequencies between 20 and $30 \mathrm{kHz}$ correspond to powerful human transmitters (groundbased) for communication with navy submarines.

The lower panel of Fig. 9 shows the integrated power at the frequency $\mathrm{f}=24.4 \mathrm{kHz}$ which is the operating frequency of the U.S. naval transmitter at Cutler, Maine. The integral power is plotted at each radial distance ( $L$ value) from $L=1.0$ to $L=4.0$. As is clear from Fig. 9b, the VLF "bubble" extends out to $L=2.8$. The integrated power at $24.4 \mathrm{kHz}$ falls off by a factor of $10^{5}$ right at the location of the impenetrable barrier identified in the REPT data.

In their work, Foster et al. $(2016,2020)$ have examined the ways in which the VLF waves inside the "bubble" could act to scatter or inhibit the local acceleration of high-energy $(\mathrm{E} \gtrsim 1 \mathrm{MeV})$ electrons, thereby greatly inhibiting such electrons from diffusing closer to the Earth than $L \sim 2.8$. While more work needs to be done to reach full understanding (including the relative importance of multiple processes acting in this region), it seems clear that human radio transmitters are playing a role in the remarkable, sharply delineated inner edge of the outer Van Allen belt (Foster et al. 2020).

\section{Summary}

Many prior published papers based on the Van Allen Probes particle and field data (including statistical studies; see Gu et al. 2020) have examined various aspects of radiation belt electron acceleration and loss processes. The goal of this present brief review paper has been to consider how certain wave modes in the magnetosphere play a key role in determining the overall structure and time variability of energetic electrons, especially those in the highly relativistic and ultra-relativistic energy domains.

Figure 10 is an adaptation of the classic diagram of Summers et al. (1998) that portrays the spatial locations of many key waves that affect the radiation belt properties. In this view-looking down onto the magnetic equatorial plane from above the Earth's north pole-one sees regions, where different wave populations are prevalent. As we have described briefly in this paper, ultra-low-frequency waves in the outer portions of the radiation belts can produce clear flux modulations and acceleration (under many conditions) as has been known for a long time (Rostoker et al. 1998). As we have noted here, ULF waves initiated by shock impacts on the dayside magnetosphere can produce rather dramatic and long-lasting effects on the relativistic electron populations at (and near) geostationary orbit altitude (see Fig. 1).

A dominant wave mode affecting radiation belt electrons are the chorus waves that are prevalent outside the 


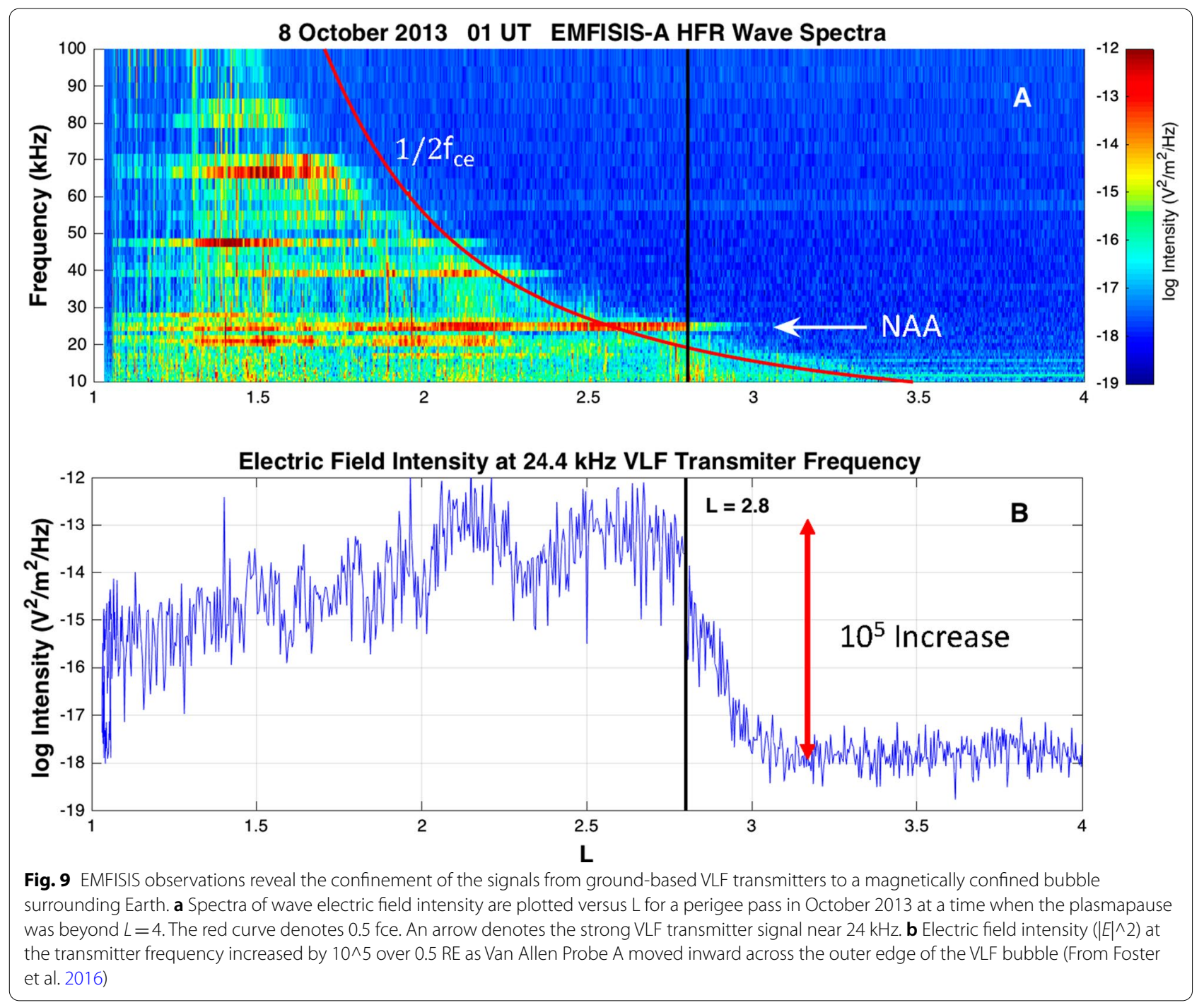

plasmasphere, especially in the post-midnight and local morning sectors. As we have described here, lower band chorus (i.e., waves a frequencies below $1 / 2$ fce) have been shown time and again to be remarkably effective at accelerating electrons throughout the outer Van Allen zone to hundreds or thousands of $\mathrm{keV}$ energies. The Van Allen Probes made major progress in detailing this chorus wave acceleration story including the role of magnetospheric substorms to provide seed particles and to amplify chorus waves.

As shown in Fig. 10, an important wave mode bridging the local noon sector is the magnetosonic wave population. Such waves outside the plasmapause produce acceleration of electron around $E=1 \mathrm{MeV}$ and generate interesting pitch angle distributions (such as those show here in Fig. 6a). The combination of lowerband chorus and magnetosonic waves can explain a good deal of the energy distributions and pitch angle properties that have been documented in Van Allen Probes statistical studies (e.g., Zhao et al. 2019b).

In the limited space available here, we were not able to address the clear effects of electromagnetic ion cyclotron (EMIC) waves on relativistic electrons deep inside the outer Van Allen zone. However, as shown by Usanova et al. (2014), Nakamura et al. (2019), EMIC waves can be shown time and again to strongly scatter off-equatorial high-energy electrons (i.e., $\alpha \neq 90^{\circ}$ ) causing rapid and effective loss of trapped electrons into the atmosphere.

Finally, plasmaspheric hiss plays a key role in the loss of hundreds of $\mathrm{keV}$ electrons in the outer zone. As reviewed here, the work of Zhao et al. (2019a) showed the previously unknown feature of a reversed relativistic electron energy spectra due to hiss interactions following geomagnetic storm events. This work has proven both qualitatively and quantitatively compelling 


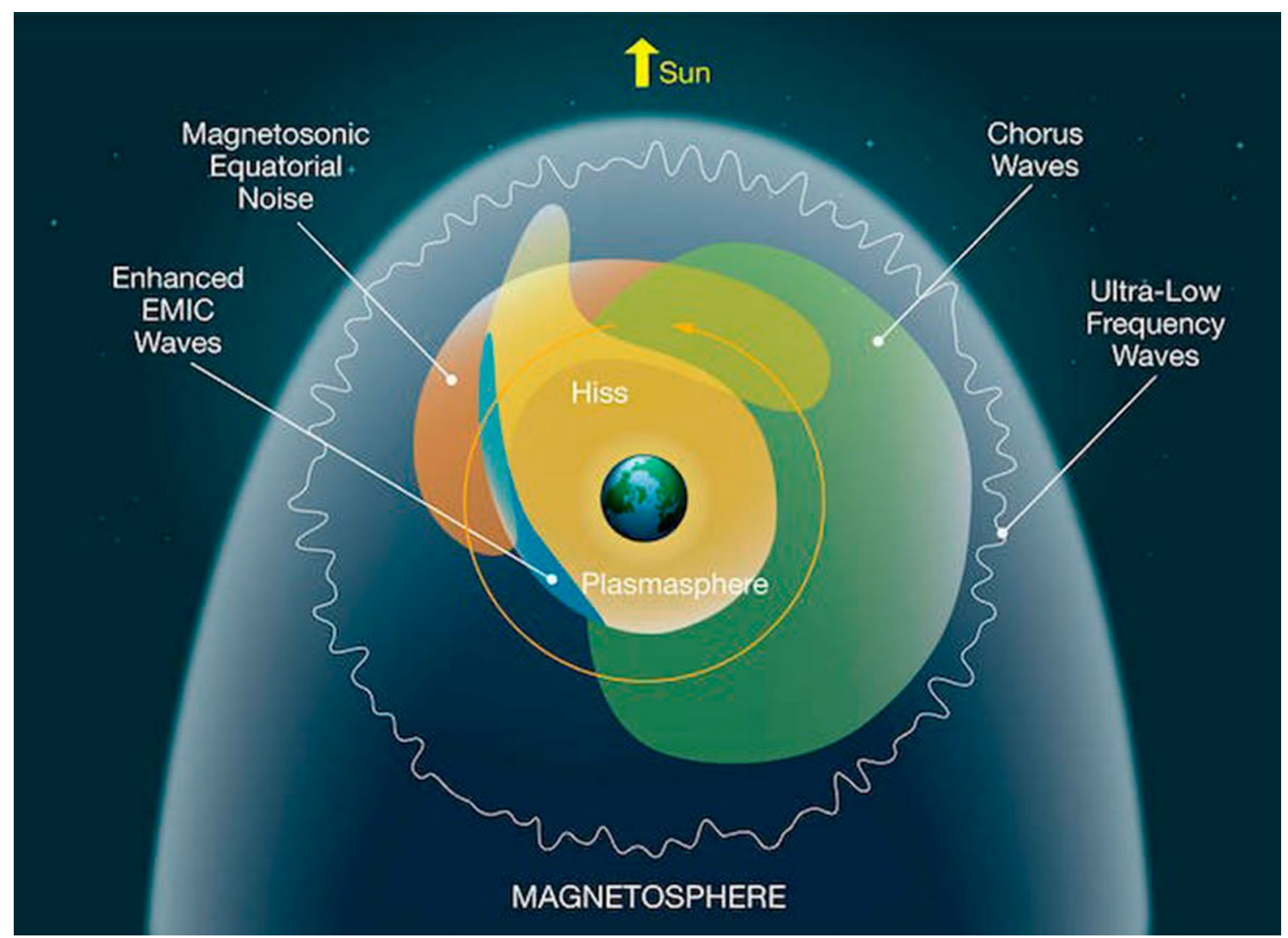

Fig. 10 Schematic diagram showing many of the wave modes that affect energetic electrons in the Earth's radiation belts (courtesy of NASA; adapted from Summers et al. 1998)

to explain new features in the high-energy electron picture for the radiation belts (see Fig. 7).

As a last point, we note that the diagram in Fig. 10 does not explicitly illustrate the VLF wave populations near Earth that we described here as the VLF "bubble" (see Figs. 8 and 9). As we have reviewed here the probable anthropogenic effects of human radio transmitters (ground-based) also need very much to be fit into the pantheon of natural wave phenomena to round out the remarkable wave-particle interaction story that the Van Allen Probes were able to tell.

\section{Acknowledgements}

I want to thank the Van Allen Probes teams and offer special thanks to John Foster and Yoshi Omura.

\section{Authors' contributions}

I am the sole author and did the work. The author read and approved the final manuscript.

\section{Funding}

The funding source is NASA Van Allen Probes. The area is space science.

\section{Availability of data and materials}

Data are available from the RBSP Gateway at JHU/APL.

\section{Declarations}

\section{Competing interests}

No competing interests.

Received: 4 June 2021 Accepted: 24 August 2021

Published online: 19 October 2021

\section{References}

Baker DN, Pulkkinen TI, Angelopoulos V, Baumjohann W, McPherron RL (1996) The neutral line model of substorms: past results and present view. J Geophys Res Space Phys 101(A6):12975-13010. https://doi.org/10.1029/ 95JA03753

Baker DN, Kanekal SG, Hoxie VC et al (2013a) The Relativistic Electron-Proton Telescope (REPT) Instrument on Board the Radiation Belt Storm Probes (RBSP) Spacecraft: characterization of Earth's Radiation Belt High-Energy Particle Populations. Space Sci Rev 179:337-381. https://doi.org/10.1007/ S11214-012-9950-9

Baker DN, Kanekal SG, Hoxie VC, Henderson MG, Li X, Spence HE, Elkington SR, Friedel RH, Goldstein J, Hudson MK, Reeves GD, Thorne RM, Kletzing CA, Claudepierre SG (2013b) A long-lived relativistic electron storage ring embedded in the Earth's outer Van Allen Radiation Zone. Science 340(6129):186-190. https://doi.org/10.1126/science.1233518

Baker DN, Jaynes AN, Li X, Henderson MG, Kanekal SG, Reeves GD, Spence HE, Claudepierre SG, Fennell JF, Hudson MK, Thorne RM, Foster JC, Erickson PJ, Malaspina DM, Wygant JR, Boyd A, Kletzing CA, Drozdov A, Shprits YY (2014a) Gradual diffusion and punctuated phase space density 
enhancements of highly relativistic electrons: Van Allen Probes observations. Geophys Res Lett 41:1351-1358. https://doi.org/10.1002/2013G L058942

Baker DN, Jaynes AN, Hoxie VC, Thorne RM, Foster JC, Li X, Fennell JF, Wygant JR, Kanekal SG, Erickson PJ, Kurth W, Li W, Ma Q, Schiller Q, Blum L, Malaspina DM, Gerrard A, Lanzerotti LJ (2014b) An impenetrable barrier to ultra-relativistic electrons in the Van Allen radiation belt. Nature 515:531-534. https://doi.org/10.1038/nature13956

Baker DN, Jaynes AN, Kanekal SG, Foster JC, Erickson PJ, Fennell JF, Blake JB, Zhao H, Li X, Elkington SR, Henderson MG, Reeves GD, Spence HE, Kletzing CA, Wygant JR (2016) Highly relativistic radiation belt electron acceleration, transport, and loss: large solar storm events of March and June 2015. J Geophys Res Space Phys. https://doi.org/10.1002/2016JA022502

Baker DN, Hoxie V, Zhao H, Jaynes AN, Kanekal S, Li X, Elkington S (2019) Multiyear measurements of radiation belt electrons: acceleration, transport, and loss. J Geophys Res Space Phys 124(4):2588-2602. https://doi.org/10. 1029/2018JA026259

Baker D, Kanekal S, Hoxie V, Li X, Jaynes A, Zhao H, ELkington S, Foster J, Selesnick R, Ni B, Spence H, Filwett R (2021) Relativistic electron-proton telescope (REPT) investigation: design, operational properties, and science highlights. Space Sci Rev. revision submitted 14 April 2021.

Blake JB et al (2013) The Magnetic Electron Ion Spectrometer (MagEIS) instruments aboard the Radiation Belt Storm Probes (RBSP) spacecraft. Space Sci Rev. https://doi.org/10.1007/s11214-013-9991-8

Foster JC, Erickson PJ, Baker DN, Claudepierre SG, Kletzing CA, Kurth W, Reeves GD, Thaller SA, Spence HE, Shprits YY, Wygant JR (2014) Prompt energization of relativistic and highly relativistic electrons during a substorm interval: Van Allen Probes observations. Geophys Res Lett 41:20-25. https://doi.org/10.1002/2013gl058438

Foster JC, Wygant JR, Hudson MK, Boyd AJ, Baker DN, Erickson PJ, Spence HE (2015) Shock-induced prompt relativistic electron acceleration in the inner magnetosphere. J Geophys Res Space Phys 120(3):1651-1674. https://doi.org/10.1002/2014ja020642

Foster JC, Erickson PJ, Baker DN, Jaynes AN, Mishin EV, Fennel JF, Li X, Henderson G, Kanekal SG (2016) Observations of the impenetrable barrier, the plasmapause, and the VLF bubble during the 17 March 2015 storm. J Geophys Res 121(6):5537-5548. https://doi.org/10.1002/2016JA022509

Foster JC, Erickson PJ, Omura Y, Baker DN, Kletzing CA, Claudepierre SG (2017) Van Allen Probes observations of prompt MeV radiation belt electron acceleration in non-linear interactions with VLF chorus. J Geophys Res Space Phys 122:324-339. https://doi.org/10.1002/2016JA023429

Foster JC, Erickson PJ, Omura Y, Baker DN (2020) The impenetrable barrier: suppression of chorus wave growth by VLF transmitters. J Geophys Res Space Phys 125:2020JA027913. https://doi.org/10.1029/2020JA027913

Gu X et al (2020) Dynamic responses of radiation belt electron fluxes to magnetic storms and their correlations with magnetospheric plasma wave activities. Astrophys J 891(2):127. https://doi.org/10.3847/1538-4357/ ab71fcJ.,891:127

Kanekal SG, Baker DN et al (2016) Near-instantaneous acceleration of magnetospheric electrons to ultra-relativistic energies by the powerful interplanetary shock during 17 March 2015. JGR Space Phys. https://doi. org/10.1002/2016JA022596

Kletzing CA, Kurth WS, Acuna M, MacDowell RJ, Torbert RB, Averkamp T et al (2013) The electric and magnetic field instrument suite and integrated science (EMFISIS) on RBSP. Space Sci Rev 179(1-4):127-181. https://doi. org/10.1007/978-1-4899-7433-4_5

Mauk BH, Fox NJ, Kanekal SG, Kessel RL, Sibeck DG, Sibeck DG, Ukhorskiy A (2013) Science objectives and rationale for the radiation belt storm probes mission. Space Sci Rev 170:3-27. https://doi.org/10.1007/ s11214-012-9908-y
Meredith $\mathrm{N}$ et al (2003) Evidence for chorus-driven electron acceleration to relativistic energies from a survey of geomagnetically disturbed periods. J Geophys Res Space Phys. https://doi.org/10.1029/2002JA009764

Miyoshi Y et al (2013) High-speed solar wind with southward interplanetary magnetic field causes relativistic electron flux enhancement of the outer radiation belt via enhanced condition of whistler waves. Geophys Res Lett 40(17):4520-4525. https://doi.org/10.1002/grl.50916

Nakamura S, Omura Y, Kletzing C, Baker DN (2019) Rapid precipitation of relativistic electron by EMIC rising-tone emissions observed by the Van Allen Probes. J Geophys Res Space Phys 124(8):6701-6714. https://doi.org/10. 1029/2019JA026772

Omura Y, Hsieh Y-K, Foster JC, Erickson PJ, Kletzing CA, Baker DN (2019) Cyclotron acceleration of relativistic electrons through landau resonance with obliquely propagating whistler mode chorus emissions. I Geophys Res Space Phys 124:2795-2810. https://doi.org/10.1029/2018JA026374

Rostoker G, Skone S, Baker DN (1998) On the origin of relativistic electrons in the magnetosphere associated with some geomagnetic storms. Geophys Res Lett 25(19):3701-3704. https://doi.org/10.1029/98GL02801

Schiller Q, Kanekal SG, Boyd AJ, Blum L, Jones AD, Baker DN, Blake JB (2017) On the cause of two prompt shock-induced relativistic electron depletion events. J Atmos Solar Terr Phys. https://doi.org/10.1016/j.jastp.2017.08. 017

Selesnick RS, Baker DN, Jaynes AN, Li X, Kanekal SG, Hudson MK, Kress BT (2016) Inward diffusion and loss of radiation belt protons. J Geophys Res Space Phys 121:1969-1978. https://doi.org/10.1002/2015JA022154

Summers D et al (1998) Relativistic theory of wave-particle resonant diffusion with application to electron acceleration in the magnetosphere. J Geophys Res Atmos 103(A9):20487-20500. https://doi.org/10.1029/98JA0 1740

Summers D et al (2007) Timescales for radiation belt electron acceleration and loss due to resonant wave-particle interactions: 2. Evaluation for VLF chorus, ELF hiss, and electromagnetic ion cyclotron waves. J Geophys Res Space Phys. https://doi.org/10.1029/2006ja011993

Usanova ME, Drozdov A, Orlova K, Mann IR, Shprits Y, Robertson MT, Turner DL, Milling DK, Kale A, Baker DN, Thaller SA, Reeves GD, Spence HE, Kletzing C, Wygant J (2014) Effect of EMIC waves on relativistic and ultra-relativistic electron populations: ground-based and Van Allen Probes observations. Geophys Res Lett 41(5):1375-1381. https://doi.org/10.1002/2013gl0590 24

Wilken B, Goertz CK, Baker DN, Higbie PR, Fritz TA (1982) The SSC on July 29, 1977 and its propagation within the magnetosphere. J Geophys Res Space Phys 87(A8):5901-5910. https://doi.org/10.1029/JA087iA08p05901

Wygant JR et al (2013) The electric field and waves (EFW) instruments on the radiation Belt Storm Probes Mission. Space Sci Rev 179:183-220. https:// doi.org/10.1007/s11214-013-0013-7

Zhao H, Ni B, Li X, Baker DN, Johnston WR, Zhang W et al (2019a) Plasmaspheric hiss waves generate a reversed energy spectrum of radiation belt electrons. Nat Phys 15(4):367-372. https://doi.org/10.1038/ s41567-018-0391-6

Zhao H, Baker DN, Li X, Malaspina DM, Jaynes AN, Kanekal SG (2019b) On the acceleration mechanism of ultrarelativistic electrons in the center of the outer radiation belt: a statistical study. J Geophys Res Space Phys 124:11. https://doi.org/10.1029/2019ja027111

\section{Publisher's Note}

Springer Nature remains neutral with regard to jurisdictional claims in published maps and institutional affiliations. 